Pure and Applied Mathematics Quarterly

Volume 1, Number 4

(Special Issue: In Memory of

Armand Borel, Part 3 of 3 )

$851-866,2005$

\title{
Remarks on the Satake Compactifications
}

\author{
Adam Korányi
}

\begin{abstract}
This article has three independent parts. The first one is a simplification, using some old results of the author, of a construction of the compactifications recently given by $\mathrm{A}$. Borel and L. Ji. The second one is another construction by directly describing the relative neighborhood systems of the boundary points. The third is a realization of the space and its compactification as a bounded domain and its closure in a vector space.
\end{abstract}

\section{INTRODUCTION}

This article is about various constructions and realizations of the Satake compactifications of Riemannian symmetric spaces. After a section on preliminaries, it consists of three logically independent parts.

The first part (Section 2) is inspired by the article of A. Borel and L. Ji on a uniform method of constructing all the known compactifications. Basic to this part are the following two observations. First, the "generalized Siegel sets" introduced and used in [1] are almost the same as the "admissible domains" introduced earlier in [10] in connection with boundary convergence of Poisson integrals on a symmetric space. Second, the proof of the "strong separation theorem" which plays an essential role in [1] can be considerably simplified by making use of the Bruhat Lemma. Based on these observations, Section 2 contains a modification of the Borel-Ji construction, making it simpler and exploiting its connections with the ideas of [10] where the possibility of a similar construction had already been sketched.

The construction of Section 2 starts with an intrinsic definition of the admissible domains, which are generalizations of non-tangential neighborhoods of the

Received September 14, 2005.

1991 Mathematics Subject Classification. 22E30, 53C35.

Partially supported by the National Science Foundation and a PSC-CUNY grant.

keywords: Riemannian symmetric spaces, Satake-Furstenberg compactifications. 
boundary points of the unit disc, then proceeds to the definition of the boundary points, and finally to the definition of the topology of the extended space. The question naturally arises whether it is possible to avoid the admisisble domains and start with giving a definition of the relative neighborhood systems of the prospective boundary points. Section 3 is devoted to showing that this can be done and leads fairly easily to a full independent construction of the Satake compactifications.

The last part, Section 4, gives realizations of symmetric spaces as bounded domains in a vector space in such a way that the ordinary closures are just the Satake compactifications. The Harish-Chandra realization of a Hermitian symmetric space is a special instance of this. The possibility of such realizations in the general case was suggested to me by the articles of L. Ji [9] and W. Casselman [3] where similar realizations are given for the maximal flat subspaces of a symmetric space. But actually the proofs in Section 4 are largely independent of [9] and [3].

I want to express my thanks to J. Faraut for his permission to use an important idea of his (for details see Section 4), and to L. Ji for many useful discussions and for the invitation to write this article.

\section{Preliminaries And Notation}

Since many of the definitions and ideas in this paper are from [10] our notations will be compatible with those of [10]. This section contains matters (sometimes slightly differently worded) that can also be found in [10]; much of this is completely standard. Some further details and references are in [10], but the present paper can be read independently.

$X \cong G / K$ will be a Riemannian symmetric space of non-compact type with $G$ connected, semi-simple and having finite center. We denote the base point of $X$, stabilized by $K$, by $o$. In $G$, we denote conjugation as $g^{h}=h g h^{-1}$ (note the difference with [1]). We will use the self-explanatory notations $U^{h}, U^{V}$ for sets $U, V$.

$\mathfrak{g}=\mathfrak{k}+\mathfrak{p}$ will be a fixed Cartan decomposition, $\theta$ the Cartan involution, $\mathfrak{a}$ a maximal abelian subspace in $\mathfrak{p}$. By roots we will always mean $\mathfrak{a}$ - roots, $\mathfrak{g}_{\lambda}$ will denote the root space corresponding to the root $\lambda$. We choose an ordering and denote by $\Pi$ the system of simple roots.

For any subset $E \subseteq \Pi$, we define the subalgebras $\mathfrak{a}(E)=\{H \in \mathfrak{a}: \lambda(H)=$ $0, \forall \lambda \in E\}$ and $\mathfrak{n}(E)=\sum \mathfrak{g}_{\lambda}$ with the sum over those $\lambda>0$ that do not vanish on $\mathfrak{a}(E)$. We define $\mathfrak{n}^{E}=\sum \mathfrak{g}_{\lambda}$ with those $\lambda>0$ that vanish on $\mathfrak{a}(E)$, and write $\mathfrak{a}^{E}$ for the orthogonal complement of $\mathfrak{a}(E)$ in $\mathfrak{a}$ with respect to the Killing form. We set $\overline{\mathfrak{n}}(E)=\theta \mathfrak{n}(E), \overline{\mathfrak{n}}^{E}=\theta \mathfrak{n}^{E}$ and we write $\mathfrak{n}, \overline{\mathfrak{n}}$ for $\mathfrak{n}(\emptyset), \overline{\mathfrak{n}}(\emptyset)$. The analytic 
subgroups of $G$ corresponding to all these subalgebras will be denoted by the corresponding Roman capitals. They are all closed. We note that $N=N^{E} N(E)$ is a semidirect product.

We let $M_{K}(E)$ be the centralizer of $\mathfrak{a}(E)$ in $K$. Then $M(E)=M_{K}(E) A^{E} N^{E}$ (also $=M_{K}(E) A^{E} \bar{N}^{E}$ ) is a reductive subgroup of $G$ given in its Iwasawa decomposition. $B(E)$, the normalizer of $N(E)$ (and of itself) is a parabolic subgroup, $B(E)=M(E) A(E) N(E)$ is its Langlands decomposition. Conjugates of $B(E)$ $(E \subseteq \Pi)$ give all the parabolic subgroups of $G$. The Lie algebras of the subgroups here introduced will be denoted $\mathfrak{m}_{K}(E), \mathfrak{m}(E), \mathfrak{b}(E)$. There is an induced Cartan decomposition $\mathfrak{m}(E)=\mathfrak{m}_{K}(E)+\mathfrak{p}^{E}$.

By the Iwasawa decomposition we have $X=G \cdot o=\bar{N} A \cdot o$, and the expression $\bar{n} a \cdot o(\bar{n} \in \bar{N}, a \in A)$ of a point is unique. (There is also a unique expression $a \bar{n}^{\prime} \cdot o$, here $\bar{n}^{\prime}=\bar{n}^{a^{-1}}$ but $a$ is the same.) Similarly we have $X^{E}=M(E) \cdot o=\bar{N}^{E} A^{E} \cdot o$, a totally geodesic subspace of $X$. Now, $X=\bar{N}(E) A(E) \cdot X^{E}$ is again a unique decomposition.

We denote by $\mathfrak{a}^{+}$the open positive Weyl chamber in $\mathfrak{a}$ and we set $\mathfrak{a}(E)^{+}=$ $\{H \in \mathfrak{a}(E): \lambda(H)>0, \forall \lambda \in \Pi \backslash E\}$ (a face of the chamber $\mathfrak{a}^{+}$). For $T \in \mathfrak{a}(E)$ we set

$$
A(E)^{T}=(\exp T) \overline{A(E)}^{+},
$$

where $A(E)^{+}=\exp \mathfrak{a}(E)^{+}$. This set can also be written $\{a \in A(E): \log a \geq T\}$ where $\log a \geq T$ means $\lambda(\log a) \geq \lambda(T)$ for all $\lambda \in \Pi \backslash E$. We say " $T$ is large in $\mathfrak{a}(E)$ ", " $\log a \rightarrow \infty$ in $\mathfrak{a}(E)$ " if $\lambda(T)$ is large, resp. $\lambda(\log a) \rightarrow \infty$ for all $\lambda \in \Pi \backslash E$.

Given $T \in \mathfrak{a}(E)^{+}$, for an element $g$ or a subset $U$ of $G$ we will write in short, $g^{T}, U^{T}$ for the sets of conjugates $A^{A(E)^{T}}, U^{A(E)^{T}}$, and will write $g^{-T}, U^{-T}$ for the sets $g^{\left(A(E)^{T}\right)^{-1}}, U^{\left(A(E)^{T}\right)^{-1}}$. It is an obvious but important fact that if $U$ is compact in $\bar{N}(E)$ then for large $T$ in $\mathfrak{a}(E)$ the set $U^{T}$ is small (it is contained in any given neighborhood of $e$ in $\bar{N}(E)$ provided $T$ is large enough). If $U$ is compact in $N(E)$, the same is true for $U^{-T}$.

For any $E \subseteq \Pi, X^{E}$ is the direct product of irreducible spaces $X^{E_{i}}$, the subsets $E_{i}$ are called the components of $E$. Let $E_{0} \subseteq \Pi$ be such that $E_{0}$ contains no component of $\Pi$. The following construction depends on $E_{0}$, which we now consider fixed.

A set $E \subseteq \Pi$ is said to be $E_{0}$-connected if no component of $E$ is contained in $E_{0}$. For such $E$, we define $E^{\prime \prime}$ as the set of all $\lambda \in E_{0}$ such that $E \cup\{\lambda\}$ is not $E_{0}$-connected, and we set $E^{\prime}=E \cup E^{\prime \prime}$. Then, $E$ is the unique maximal $E_{0^{-}}$ connected subset of $E^{\prime}$ and $E^{\prime}$ is the unique maximal subset of $\Pi$ with the latter property. $E$ and $E^{\prime \prime}$ are disjoint, both are unions of components of $E^{\prime}$. There is a corresponding direct product decomposition $X^{E^{\prime}}=X^{E} \times X^{E^{\prime \prime}}$. We have $M\left(E^{\prime}\right)=M(E) M\left(E^{\prime \prime}\right)$ and $M\left(E^{\prime}\right)$ preserves the product structure of $X^{E^{\prime}}$. So, 
$M\left(E^{\prime}\right)$ acts on $X^{E}$ by projecting its action on $X^{E^{\prime}}$. The stabilizer for this action is $M_{K}(E) M\left(E^{\prime \prime}\right)$. The group $B\left(E^{\prime}\right)=M\left(E^{\prime}\right) A\left(E^{\prime}\right) N\left(E^{\prime}\right)$ can also be made to act on $X^{E}$ by defining the action of $A\left(E^{\prime}\right) N\left(E^{\prime}\right)$ to be trivial. We denote the stabilizer of $o$ for this action by $B_{\mathrm{st}}\left(E^{\prime}\right)$. We have $B_{\mathrm{st}}\left(E^{\prime}\right)=M_{K}(E) M\left(E^{\prime \prime}\right) A\left(E^{\prime}\right) N\left(E^{\prime}\right)$. This is the same group as in Remark 3 on page 22 of [10]; it will play an important role later on.

To each one of the subsets $E_{0}$ there belongs a Satake compactification $\bar{X}\left(E_{0}\right)$. These have axiomatic characterizations due to Satake ([13], or rewritten in our present notations, [10, pp. 21-22]). There are a number of ways of constructing them, i.e. to prove their existence (e.g. [13], [12], [6], [9], [1]). The present paper will add a few more.

\section{A COnstruction of the COMPACtifications}

In order to avoid many repetitions we make the following conventions. For any subgroup $H \subseteq G$ or any one of the spaces $X^{E}$, the notations $V_{H}, V_{X^{E}}$ will automatically mean compact neighborhoods of $e$ in $H$, resp. of $o$ in $X^{E}$. Similarly, $U_{H}, U_{X^{E}}$ will mean compact subsets of $H$ or $X^{E}$.

Throughout this section we will be in the situation described in Section 1. A subset $E_{0} \subseteq \Pi$ will be given once and for all, $E$ will always stand for an $E_{0}$ connected set, $E^{\prime}, E^{\prime \prime}$ etc. will be as in Section 1.

Definition 2.1. For $T \in \mathfrak{a}\left(E^{\prime}\right), U=U_{\bar{N}\left(E^{\prime}\right)}, C=U_{M\left(E^{\prime \prime}\right)}, V=V_{M\left(E^{\prime}\right)}$ we set

$$
\Gamma_{U, C}^{T, V}=A\left(E^{\prime}\right)^{T} U C V \cdot o
$$

These sets were introduced in [10] and were called admissible domains. they are generalizations of non-tangential angular neighborhoods of the point 1 in the complex unit disc, $U$ and $C$ playing the role of the opening angle. They and their translates by elements of $G$ are used in [10] to prove a generalization of Fatou's theorem about non-tangential limits of harmonic functions.

Since $U, C, V$ are compact and all elements of $G$ are isometries, $\Gamma_{U, C}^{T, V}$ is a set of points within a bounded distance of $A\left(E^{\prime}\right)^{T} \cdot o$ (which is a polyhedral cone in the flat subspace $A\left(E^{\prime}\right) \cdot o$ of $X$, "far out at infinity" if $T$ is large). This is also the crucial feature of the generalized Siegel sets $S_{\varepsilon, T, V}$ introduced by Borel and Ji [1]. What we want to show is that the Borel-Ji construction can be simplified by replacing the $S_{\varepsilon, T, V}$ by the $\Gamma_{U, C}^{T, V}$ and using some new arguments along part of the way.

The following is a slight strengthening of [10, Lemma 2.1]. 
Lemma 2.2. Let $\tilde{U}=U_{B_{\mathrm{st}}\left(E^{\prime}\right)}$ be given. Then for any $U, C$ as above there exists $U_{1}, C_{1}$ such that for any $T_{1}, V_{1}$ there can be found $T, V$ with the property $\tilde{U} \Gamma_{U, C}^{T, V} \subseteq$ $\Gamma_{U_{1}, C_{1}}^{T_{1}, V_{1}}$

Proof. It suffices to consider the four cases $\tilde{U} \subseteq A\left(E^{\prime}\right), \tilde{U} \subseteq M\left(E^{\prime \prime}\right), \tilde{U} \subseteq M_{K}(E)$, $\tilde{U} \subseteq N\left(E^{\prime}\right)$. The first case is trivial: we may take $U_{1}=U, C_{1}=C$, and then $V=V_{1}, T=T^{1}+T_{0}$ where $-T_{0}$ is a "lower bound" for $\tilde{U}$, that is, $\tilde{U} \subseteq A\left(E^{\prime}\right)^{-T_{0}}$. In the second case

$$
\tilde{U} \Gamma_{U, C}^{T, V}=A\left(E^{\prime}\right)^{T} U^{\tilde{U}}(\tilde{U} C) V \cdot o,
$$

so $U_{1}=U^{\tilde{U}}, C_{1}=\tilde{U} C$ will work (with $T=T_{1}, V=V_{1}$ ). In the third case,

$$
\tilde{U} \Gamma_{U, C}^{T, V}=A\left(E^{\prime}\right)^{T} U^{\tilde{U}} C^{\tilde{U}} V^{\tilde{U}} \cdot o
$$

and the situation is again clear.

Finally, if $\tilde{U} \subseteq N\left(E^{\prime}\right)$, first we fix some $\tilde{T}$ and $\tilde{V}$. For large $T, \tilde{U}^{-T} U C V \cdot o$ is a small neighborhood in $X$ of the set $U C V \cdot o$. Therefore (using $\tilde{U} A\left(E^{\prime}\right)^{T}=$ $\left.A\left(E^{\prime}\right)^{T} \tilde{U}^{-T}\right)$ we can fix $U_{1}, C_{1}$ so that

$$
\tilde{U} \Gamma_{U, C}^{T, V} \subseteq \tilde{U} \Gamma_{U_{1}, C_{1}}^{\tilde{T}, \tilde{V}}
$$

holds for all sufficiently large $T$ and small $V$. With this $U_{1}, C_{1}$, the assertion of the Lemma holds: for any $V_{1}$ there is $T$ large enough so that $\tilde{U}^{-T} U C V \cdot o \subseteq$ $U_{1} C_{1} V_{1} \cdot o$.

The following is our version of the "strong separation" property, essential in the Borel-Ji construction. We will consider two $E_{0^{-}}$connected sets $E, E_{1}$ and corresponding admissible domains. Sets without subscripts correspond to $E$, sets with subscript 1 to $E_{1}$

Proposition 2.3. If $E \neq E_{1}$ or if $E=E_{1}$ and $g \notin B_{\mathrm{st}}\left(E^{\prime}\right)$, then for any $U$, $C, U_{1}, C_{1}$, there exist $T, V, T_{1}, V_{1}$ and $V_{G}$ such that $g \Gamma_{U, C}^{T, V}$ and $V_{G} \Gamma_{U_{1}, C_{1}}^{T_{1}, V_{1}}$ are disjoint.

Proof. First we note that instead of $V_{G}$ it is enough to find $V_{\bar{N}\left(E_{1}^{\prime}\right)} V_{M\left(E_{1}^{\prime}\right)}$ with the same property. In fact, we can fix an (arbitrary) $\tilde{V}=V_{B_{\mathrm{st}}\left(E_{1}^{\prime}\right)}$ and by Lemma 2.2 find $U_{2} \supset U_{1}, C_{2} \supset C_{1}$ so that $\tilde{V} \Gamma_{U_{2}, C_{2}}^{T_{2}, V_{2}} \subseteq \Gamma_{U_{1}, C_{1}}^{T_{1}, V_{1}}$ for large $T_{2}$ and small $V_{2}$. Then $V_{G}=\tilde{V} V_{\bar{N}\left(E_{1}^{\prime}\right)} V_{M\left(E_{1}^{\prime}\right)}$ will have the required property.

We express $g$ with the aid of the Bruhat decomposition $G=\cup_{w} \bar{N}\left(E^{\prime}\right) w M\left(E^{\prime}\right) A\left(E^{\prime}\right) N\left(E^{\prime}\right)$; here $w \in W / W^{E^{\prime}}$ with $W^{E^{\prime}}$ denoting the subgroup of the Weyl group $W$ generated by reflections coming from $M\left(E^{\prime}\right)$. $w$ is represented by an element of $M^{\prime}$, the normalizer of $\mathfrak{a}$ in $K$. Again, by Lemma 2.2, the $A\left(E^{\prime}\right) N\left(E^{\prime}\right)$ - part of $g$ will 
be irrelevant for our statement, so we may assume that $g=\bar{n} w m\left(\bar{n} \in \bar{N}\left(E^{\prime}\right)\right)$, $\left.m \in M\left(E^{\prime}\right)\right)$. Now we have

$$
g \Gamma_{U, C}^{T, V}=\bar{n}\left(A\left(E^{\prime}\right)^{T}\right)^{w} U^{w m} C^{w m} V^{w m} w m \cdot o
$$

and the claim is that for appropriate choices this is disjoint from

$$
V_{\bar{N}\left(E_{1}^{\prime}\right)} V_{M\left(E_{1}^{\prime}\right)} A\left(E_{1}^{\prime}\right)^{T_{1}} U_{1} C_{1} V_{1} \cdot o=V_{\bar{N}\left(E_{1}^{\prime}\right)} A\left(E_{1}^{\prime}\right)^{T_{1}} \tilde{U}_{1} V_{M\left(E_{1}^{\prime}\right)} C_{1} V_{1} \cdot o
$$

where $\tilde{U}_{1}$ is still in $\bar{N}\left(E_{1}^{\prime}\right)$, since $M\left(E_{1}^{\prime}\right)$ normalizes $N\left(E_{1}^{\prime}\right)$. In both sets we write the points in the form $\bar{n} a \cdot o$ using the unique decomposition $X=\bar{N} A \cdot o$. The first step in this is to write the points of $U^{w m} C^{w m} V^{w m} w m \cdot o$ and of $\tilde{U}_{1} V_{M\left(E_{1}^{\prime}\right)} C_{1} V_{1} \cdot o$ in such a form. These sets being compact, the $A$ - components we obtain are bounded. If $E \neq E_{1}$, or $w \neq e$, the Weyl chamber faces $\left(\mathfrak{a}\left(E^{\prime}\right)^{+}\right)^{w}$ and $\mathfrak{a}(E)^{+}$are different. Hence taking $T$ and $T_{1}$ large we can make sure that the $A$-components of all elements of (2.2) and (2.3) are different, so these sets are disjoint.

If $E=E_{1}$ and $w=e$, but $\bar{n} \neq e$ then in (2.2) and (2.3) we have $U^{w m} \subseteq \bar{N}\left(E^{\prime}\right)$, furthermore, $C^{m w} V^{m w} m w \cdot o$ and $V_{M\left(E_{1}^{\prime}\right)} C_{1} V_{1} \cdot o$ are in $X^{E^{\prime}}$. If we write the elements of (2.2), (2.3) in the decomposition $X=\bar{N}\left(E^{\prime}\right) A\left(E^{\prime}\right) \cdot X^{E^{\prime}}$, the $\bar{N}\left(E^{\prime}\right)$ component will be in $\bar{n}\left(U^{w m}\right)^{T}$, resp. in $V_{\bar{N}\left(E_{1}^{\prime}\right)} \tilde{U}_{1}^{T_{1}}$ So, when $\bar{n} \notin V_{\bar{N}\left(E_{1}^{\prime}\right)}$ and $T, T_{1}$ are large, the two sets are disjoint.

Finally, if $E=E_{1}, w=e$ and $\bar{n}=e$, then the hypothesis implies $g=m \notin$ $M_{K}(E) M\left(E^{\prime \prime}\right)$. Taking $V_{M\left(E_{1}^{\prime}\right)}$ such that $e \notin V_{M\left(E_{1}^{\prime}\right)},(2.2)$ and (2.3) will again be disjoint for large $T, T_{1}$ and small $V, V_{1}$.

In order to compare with the Borel-Ji article we reformulate and slightly extend the fundamental Proposition 2.4 of [1] in our language:

If $E \neq E_{1}$ or $E=E_{1}$ and $g \notin B(E)$, then for any $U_{X}, g A(E)^{T} \cdot U_{X}$ and $V_{G} A\left(E_{1}\right)^{T_{1}} \cdot U_{X}$ are disjoint for large $T, T_{1}$, provided $g^{-1} V_{G}$ is disjoint from $B(E)$.

Our argument above, with unessential modifications, proves this statement. It also proves Proposition 4.1 in [1], which is a complement to Proposition 2.4 dealing with the case $E=E_{1}, g \in B(E)$. Our argument can be said to be simpler since it does not make use of Satake's idea of imbedding $X$ into a projective space; it is also shorter. As a curiosity we might mention that there is yet another possible approach to this question: One can use the results of [10] about convergence of the Poisson integral along the sets $\Gamma_{U, C}^{T, V}$ to any given continuous boundary function to prove the desired separation. Such a proof, in the last analysis, is based on the Furstenberg-Moore embedding [5], [12] of $X$ into a space of probability measures, just as the proof in [1] is based on Satake's imbedding.

In any case, having proposed an alternative proof of Propositions 2.4 and 4.1 of [1], we could say that the rest of the construction proceeds as in [1]. But we 
want to make some further remarks about combining the ideas of [1] with those of [10] in order to arrive at a construction which is perhaps the most natural one.

As in [10], for fixed $E, U, C$ we consider the filter $\mathcal{F}_{U, C}^{E}$ generated by all $\Gamma_{U, C}^{T, V}$. We denote by $g \mathcal{F}_{U, C}^{E}$ the filter of the $g$-translates $g \Gamma_{U, C}^{T, V}$ and by $g \tilde{\mathcal{F}}^{E}$ the family of all filters $g \mathcal{F}_{U, C}^{E}$. We say that two families are equivalent if for every filter in one of the families there is a rougher one in the other. (This means that if a sequence converges along some filter in one family then it also converges along some filter in the other; in the present case this is generalized non-tangential convergence.) The points of the compactification $\bar{X}\left(E_{0}\right)$ will now be the equivalence classes of filters $g \tilde{\mathcal{F}}^{E}$. In this way the points to be attached to $X$ appear together with ways to tend towards them from the interior.

Lemma 2.2 and Proposition 2.3 show that $g \tilde{\mathcal{F}}^{E}$ is equivalent to $\tilde{\mathcal{F}}^{E}$ if and only if $g \in B_{\mathrm{st}}\left(E^{\prime}\right)$. One can write $g \cdot \iota_{E}(o)$ for $g \tilde{\mathcal{F}}^{E}$ and define the imbedding $\iota_{E}$ of $X^{E}$ into $\bar{X}\left(E_{0}\right)$ by $\iota_{E}(m \cdot o)=m \cdot \iota_{E}(o)$ for $m \in M(E)$. Then $\bar{X}\left(E_{0}\right)$ is disjoint union of "boundary components" $g \cdot \iota_{E}\left(X^{E}\right)$, and one has a complete description of $\bar{X}\left(E_{0}\right)$ as a $G$-set.

It remains to define the topology of $\bar{X}\left(E_{0}\right)$ and prove its properties. At this point we can say that the construction proceeds as in [1], by describing the class of convergent sequences and verifying the required properties. We should remark here also that a convenient neighborhood basis of $\iota_{E}(o)$ can be obtained as follows. A basis of relative neighborhoods with respect to $X$ of $\iota_{E}(o)$ is formed by the sets

$$
V_{G} M_{K}\left(E^{\prime \prime}\right) A(E)^{T^{\prime}} \cdot o
$$

with $T^{\prime} \in \mathfrak{a}\left(E^{\prime}\right)$. ( $\left(T^{\prime}\right.$ is restricted to the subset $\mathfrak{a}\left(E^{\prime}\right)$ of $\mathfrak{a}(E)$.) It is not hard to see that this description is equivalent to the one given in Section 5 of [1]. Similarly, full neighborhoods of $\iota_{E}(o)$ are unions over $E_{0}$-connected sets $D \supset E$ :

$$
V_{G} M_{K}\left(E^{\prime \prime}\right)\left(\cup A^{D}(E)^{T_{D}^{\prime}} \cdot \iota_{D}(o)\right)
$$

where $T_{D}^{\prime} \in \mathfrak{a}\left(D^{\prime}\right)$.

\section{Another Construction}

In this section, which is independent of the preceding one, we describe another construction of the Satake compactifications. This will still be "from inside", that is, by attaching points to $X$, but without the use of Siegel domains or admissible domains. We will directly define the system of relative neighborhoods of the points to be attached, thus obtaining $\bar{X}\left(E_{0}\right)$ as a set. Then we will define the action of $G$, show that the relative closures of the relative neighborhoods give the full neighborhood system of a topology, and then verify that what we have constructed is a really the Satake compactification. 
In order not to get too lengthy and complicated, we will carry this out in detail only for the maximal compactification $\bar{X}=\bar{X}(\emptyset)$. We will then briefly sketch the modifications and additional arguments necessary for the general case.

Definition 3.1. For $E \subseteq \Pi$, let $\mathcal{F}^{E}$ be the filter generated by the sets $V_{G} A(E)^{T}$.o $\left(V_{G} \subset G, T \in \mathfrak{a}(E)\right)$.

REMARK 1. The filters $\mathcal{F}_{U}^{E}$ of Section 2 (now without $C$, and with $E^{\prime}=E$, since $\left.E_{0}=\emptyset\right)$ are all finer than $\mathcal{F}^{E}$. In fact, given $V_{G}$ and $T$, for any $U$ there exist $\tilde{T}, V$ such that $\Gamma_{U}^{\tilde{T}, V}=A(E)^{\tilde{T}} U V \cdot o=U^{\tilde{T}} V A(E)^{\tilde{T}} \cdot o \subseteq V_{G} A(E)^{T} \cdot o$.

Lemma 3.2. The sets $V_{\bar{N}(E)} A(E)^{T} \cdot V_{X^{E}}$ or $V_{\bar{N} A^{E}} A(E)^{T}$. o also generate $\mathcal{F}^{E}$.

Proof. The sets written are the same by $\bar{N}=\bar{N}(E) \bar{N}^{E}$ and $X^{E}=\bar{N}^{E} A^{E} \cdot o$. We must show that for given $\tilde{V}_{\bar{N}(E)} A(E)^{\tilde{T}} \cdot V_{X^{E}}$ there exists some $V_{G} A(E)^{T} \cdot o$ contained in it. We look for $V_{G}$ in the form

$$
V_{G}=V_{\bar{N}(E)} V_{N(E)} V_{A(E)} V_{M(E)} .
$$

We choose $V_{N(E)}$ and $V_{A(E)}$ arbitrarily and $V_{M(E)}, V_{\bar{N}(E)}$ such that

$$
\begin{gathered}
\tilde{V}_{X^{E}}=V_{M(E)} \cdot o \Subset V_{X^{E}} . \\
V_{\bar{N}(E)} \Subset \tilde{V}_{\bar{N}(E)} .
\end{gathered}
$$

(The notation $\Subset$ means "contained in the interior of".) We take $T_{0} \in \mathfrak{a}^{+}(E)$ such that $V_{A(E)} \subseteq A(E)^{-T_{0}}$.

For any choice of $\tilde{\tilde{V}}_{\bar{N}(E)}$ we will have, for sufficiently large $T$,

$$
V_{N(E)}^{-\left(T-T_{0}\right)} \cdot \tilde{V}_{X^{E}} \subset V_{A(E)} \tilde{\tilde{V}}_{\bar{N}(E)} \cdot V_{X^{E}}
$$

because the right hand side is a neighborhood in $X$ of the compact set $\tilde{V}_{X^{E}}$.

It follows that for sufficiently large $T$,

$$
\begin{aligned}
V_{\bar{N}(E)} V_{N(E)} V_{A(E)} V_{M(E)} A(E)^{T} \cdot o & \subseteq V_{\bar{N}(E)} A(E)^{T-T_{0}} V_{N(E)}^{-\left(T-T_{0}\right)} \tilde{V}_{X^{E}} \\
& \subseteq V_{\bar{N}(E)} \tilde{\tilde{V}}_{\bar{N}(E)}^{T-2 T_{0}} A(E)^{T-2 T_{0}} \cdot V_{X^{E}} \\
& \subseteq \tilde{V}_{\bar{N}(E)} A(E)^{\tilde{T}} \cdot V_{X^{E}}
\end{aligned}
$$

finishing the proof.

Lemma 3.3. Given $U=U_{\mathrm{B}_{\mathrm{st}}(\mathrm{E})}$ and any $\tilde{V}_{G}, \tilde{T} \in \mathfrak{a}(E)$, there exist $V_{G}, T$ such that

$$
U V_{G} A(E)^{T} \cdot o \subset \tilde{V}_{G} A(E)^{\tilde{T}} \cdot o
$$


Proof. It suffices to fix some $\tilde{\tilde{V}}_{G} \Subset \tilde{V}_{G}$ and find $T$ such that

$$
U A(E)^{T} \cdot o \subseteq \tilde{\tilde{V}}_{G} A(E)^{\tilde{T}} \cdot o
$$

because then we can always find $V_{G}$ such that $V_{G}^{U} \tilde{\tilde{V}}_{G} \subseteq \tilde{V}_{G}$, and (3.1) follows.

To prove (3.2) we distinguish cases. When $U \subseteq M_{K}(E)$, the statement is trivial since $M_{K}(E)$ commutes with $A(E)$ and fixes $o$. The case $U \subseteq A(E)$ is also trivial: then $U A(E)^{T} \subseteq A(E)^{T+T_{0}}$ with some $T_{0} \in \mathfrak{a}(E)$. When $\bar{U} \subseteq N(E)$, we choose some $V_{\bar{N}(E)} V_{M(E)} \subseteq \tilde{\tilde{V}}_{G}$ and some arbitrary $V_{A(E)}$. For large $T$ we have

$$
U^{-T} \cdot o \subseteq V_{\bar{N}(E)} V_{M(E)} V_{A(E)} \cdot o
$$

since the right side is a neighborhood of $o$ in $X$. So, for large $T$,

$$
\begin{aligned}
U A(E)^{T} \cdot o & =A(E)^{T} U^{-T} \cdot o \\
& \subseteq A(E)^{T} V_{\bar{N}(E)} V_{M(E)} V_{A(E)} \cdot o \\
& =V_{\bar{N}(E)}^{T} V_{M(E)} A(E)^{T} V_{A(E)} \cdot o \\
& \subseteq \tilde{\tilde{V}}_{G} A(E)^{\tilde{T}} \cdot o
\end{aligned}
$$

as was to be shown.

One consequence of this Lemma is that $g \mathcal{F}^{E}=\mathcal{F}^{E}$ if $g \in B_{\text {st }}(E)$. (By $g \mathcal{F}^{E}$ we mean the filter formed by the sets $g S, S \in \mathcal{F}^{E}$.) Writing the generators of $\mathcal{F}^{E}$ as in Lemma 3.2 it is immediate that this is actually "if and only if". We can now define $\bar{X}=\bar{X}(\emptyset)$ as a set by attaching all filters $g \mathcal{F}^{E}(g \in G, E \subseteq \Pi)$ to $X$; more intuitively we can say that the filter $g \mathcal{F}^{E}$ determines a point $g \cdot \iota_{E}(o)$ of $\bar{X}$. We can define the imbedding $\iota_{E}: X^{E} \rightarrow \bar{X}$ consistently by setting $\iota_{E}(m \cdot o)=m \cdot \iota_{E}(o)$ for $m \in M(E)$. It is then easy to see that each orbit $G \cdot \iota_{E}(o)$ is the disjoint union of boundary components of the form $g \cdot \iota_{E}\left(X^{E}\right)$, the family of these being parametrized by $G / B(E)$.

To complete the construction of $\bar{X}$ we must define its topology. We want this to be such that $g \mathcal{F}^{E}$ is exactly the system of relative $X$-neighborhoods of $g \cdot \iota_{B}(o)$. The full neighborhoods then must include the closures of the elements of $g \mathcal{F}^{E}$.

The closure of an element $\mathcal{N} \in \mathcal{F}^{E}$ must contain the relative accumulation points, that is, those $g \cdot \iota_{D}(o) \in \bar{X}$ for which every element of $g \mathcal{F}^{D}$ meets $\mathcal{N}$. We proceed to determine these for a family of generators of $\mathcal{F}^{E}$.

We denote by $\operatorname{pr}^{D}$ the projection of $\mathfrak{a}(E)$ onto $\mathfrak{a}^{D}(E)$ (which is the joint 0-space of $E$ in $\mathfrak{a}^{D}$ ) along the decomposition $\mathfrak{a}^{D}(E) \oplus \mathfrak{a}(D)$.

Proposition 3.4. The relative accumulation points of $\mathcal{N}=V_{\bar{N} A_{E}} A(E)^{T} \cdot o$ are the points $g \cdot \iota_{D}(o)$ with $D \supset E, g \in V_{\bar{N} A^{E}} A^{D}(E)^{\operatorname{pr}^{D} T}$. 
Proof. For any $D \subseteq \Pi$ we have

$$
G=\cup_{w \in W / W^{D}} \bar{N}(D) w B(D)
$$

by the Bruhat Lemma. Together with Lemma 3.3 this shows that all the points of $\bar{X}=\bar{X}(\emptyset)$ can be uniquely written as $g \cdot \iota_{D}(o)$ with $g=\bar{n}_{D} w \bar{n}^{D} a^{D}\left(\bar{n}_{D} \in \bar{N}(D)\right.$, $\left.w \in W / W^{D}, \bar{n}^{D} \in \bar{N}^{D}, a^{D} \in A^{D}\right)$. We must determine for which ones of these does $g \tilde{\mathcal{N}}=g \tilde{V}_{\bar{N} A^{D}} A(D)^{\tilde{T}} \cdot o$ meet $\mathcal{N}$ for all $\tilde{V}_{\bar{N} A^{D}}$ and all $\tilde{T} \in \mathfrak{a}(D)$.

We consider first the case where $w=e$. If $E \nsubseteq D$, there is a root $\lambda \in E \backslash D$. For $\bar{n} a \cdot o \in g \tilde{\mathcal{N}}$ (with $\bar{n} \in \bar{N}, a \in A$ ), then $\lambda(\log a)$ will be very large for large $\tilde{T}$ while $\lambda(\log a)$ is bounded on $\mathcal{N}$. So, $\mathcal{N}$ can't meet every $g \tilde{\mathcal{N}}$.

Suppose now that $D \supset E$. Then we can rewrite $g$ as $g=\bar{n} a^{E} a_{E}^{D}$ (with $\left.\bar{n}=\bar{n}_{D} \bar{n}^{D}, a^{D}=a^{E} a_{E}^{D}\right)$ using the decomposition $A^{D}=A^{E} A^{D}(E)$.

If $g \in V_{\bar{N} A_{\tilde{N}}} A^{D}(E)^{\operatorname{pr}^{D} T} \cdot o$ then $\bar{n} a^{E} \in V_{\bar{N} A^{E}}$ and $\log a_{E}^{D} \geq \operatorname{pr}^{D} T$ in $\mathfrak{a}^{D}(E)$. Taking any $\tilde{\mathcal{N}}$, the set $g \tilde{\mathcal{N}}$ contains $\bar{n} a^{E} a_{E}^{D} \tilde{a}_{D} \cdot o$ for all suffciently large $\tilde{a}_{D}$ in $A(D)$. But among these there are points which are also in $\mathcal{N}$. Indeed, for $\lambda \in D \backslash E$ we have $\lambda\left(\log \left(a_{E}^{D} \tilde{a}_{D}\right)\right)=\lambda\left(\log a_{E}^{D}\right) \geq \lambda\left(\operatorname{pr}^{D} T\right)=\lambda(T)$, and for $\lambda \in \Pi \backslash D, \lambda\left(\log \left(a_{E}^{D} \tilde{a}_{D}\right)\right)=\lambda\left(\log a_{E}^{D}\right)+\lambda\left(\log \tilde{a}_{D}\right)$ will be larger than $\lambda(T)$ for large $\tilde{a}_{D}$, showing that $a_{E}^{D} \tilde{a}_{D} \in A(E)^{T}$.

In the contrary case either $\bar{n} a^{E} \notin V_{\bar{N} A^{E}}$ or $\log a_{E}^{D} \nsucceq \operatorname{pr}^{D} T$. In either case, for sufficiently small $\tilde{V}_{\bar{N} A^{D}}, g \tilde{\mathcal{N}}$ will be disjoint from $\mathcal{N}$, as can be seen by looking at the $\bar{N} A^{E}$ - part resp. the $A^{D}(E)$ - part in the unique representation of $X$ as $\bar{N} A \cdot 0$.

Now we consider the case where $w \neq e$ in $W / W^{D}$. So, now $g=\bar{n}_{D} w \bar{n}^{D} a^{D}$ $\left(w \notin W^{D}\right)$ and we ask the question: Is there such a $g$ with the property that for all $\tilde{V}_{G}, \tilde{T}$, the set $g \tilde{\mathcal{N}}=g \tilde{V}_{G} A(D)^{\tilde{T}} \cdot o$ meets $\mathcal{N}$ ?

Since $g \tilde{V}_{G}=\tilde{V}_{G}^{g} g$ the question amounts to whether $g A(D)^{\tilde{T}} \cdot o$ meets $\left(\tilde{V}_{G}^{g}\right)^{-1} \mathcal{N}$ for all $\tilde{V}_{G}, \tilde{T}$. The first one of these sets can be written as $\bar{n}_{D}\left(A(D)^{\tilde{T}}\right)^{w} U_{\bar{N} A} \cdot o$. For the second set we can use Lemma 3.3 to see that, for small $\tilde{V}_{G}$, it is contained in some $\tilde{U}_{\tilde{N} A} A(E)^{\tilde{\tilde{T}}} \cdot o$. Writing the points of $X$ in the form $\bar{n} a \cdot o(\bar{n} \in \bar{N}, a \in A)$ we see that on the second set $\lambda(\log a)$ is bounded below for every $\lambda \in \Pi$. Since $w \notin W^{D}$, some $\lambda$ will be negative on $\left(\mathfrak{a}(D)^{+}\right)^{w}$. Hence for large $\tilde{T}, \lambda(\log a)$ will be negative with large absolute value on the first set. It follows that for large $\tilde{T}$ there is no intersection.

By this Proposition if we attach to $\mathcal{N}$ its relative accumulation points, we obtain the set

$$
V_{\bar{N} A^{E}} \cdot\left(\cup_{D \supset E} \iota_{D}\left(A^{D}(E)^{\operatorname{pr}^{D} T} \cdot o\right)\right) .
$$


Every neighborhood of $\iota_{E}(0)$ in the topology we are trying to construct must contain such a set. Now it is possible to check that these sets and their translates by elements of $G$ already form the basis of a topology which we then define to be the topology of $\bar{X}(\emptyset)$. One way to do this checking is to verify that the "closure" $\bar{S}$ of sets $S \subseteq \bar{X}(\emptyset)$ determined by our prospective neighborhoods (3.3) (and their translates) satisfies the Kuratowski axioms: $\bar{\emptyset}=\emptyset, S \subseteq \bar{S}, \overline{\bar{S}}=\bar{S}, \overline{S \cup T}=\bar{S} \cup \bar{T}$.

It is immediate that in (3.3) we can replace $V_{\bar{N} A^{E}}$ by $V_{G}$. The continuity of the $G$-action follows from Lemma 3.3. From the axiomatic characterization of the compactification ([13], or rewritten in the present notation [10, pp. 22-23]) it is clear that the topology we have constructed is indeed the Satake topology. This can also be seen by comparing (3.3) with the neighborhood basis given in Section 4 of [1].

Now, to finish, we describe the modifications required for the general case, that is, for constructing the Satake compactifications $\bar{X}\left(E_{0}\right)$. In this case, we take only $E_{0}$-connected sets $E$, and define $\mathcal{F}^{E}$ to be the filter generated by the sets

$$
V_{G} M_{K}\left(E^{\prime \prime}\right) A(E)^{T^{\prime}} \cdot o
$$

for all $V_{G}$ and all $T^{\prime} \in \mathfrak{a}\left(E^{\prime}\right)$. (Note the restriction on $T^{\prime}$.) The general version of Lemma 3.2 says now that $\mathcal{F}^{E}$ is also generated by the sets $V_{\bar{N}}\left(E^{\prime}\right) \bar{N}^{E} A^{E} M_{K}\left(E^{\prime \prime}\right)$ $A(E)^{T^{\prime}} \cdot o$ or $V_{\bar{N}\left(E^{\prime}\right)} M_{K}\left(E^{\prime \prime}\right) A(E)^{T^{\prime}} \cdot V_{X^{E}}$. Lemma 3.3 remains true with $U=$ $U_{B_{\mathrm{st}}\left(E^{\prime}\right)}$ for the generators of $\mathcal{F}^{E}$; in the proof one has to split the $A(E)^{T^{\prime}}$ component into factors in $A\left(E^{\prime}\right)$ and $A^{E^{\prime \prime}}$ and use the Cartan decomposition of $M_{K}\left(E^{\prime \prime}\right)$. One defines the set $\bar{X}\left(E_{0}\right)$ and imbeddings $\iota_{E}$ as in the special case $E_{0}=\emptyset$. Proposition 3.4 now says that the relative accumulation points of

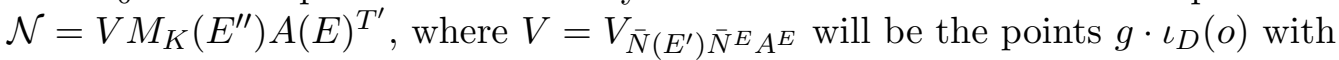
$E_{0}$-connected $D \supset E$ and $g \in V M_{K}\left(E^{\prime \prime}\right) A^{D}(E)^{\mathrm{pr}^{D} T^{\prime}}$. The construction of the topology then proceeds as in the case $E_{0}=\emptyset$.

\section{Bounded DOMAin REALizations}

In this section, which is independent of the preceding two, we will give realizations of $X \cong G / K$ as bounded domains in $\mathfrak{p}$ in such a way that the Satake compactifications can be obtained by taking the ordinary closure in $\mathfrak{p}$. Many of the elements needed for this result are in [9], [3], [6, Ch. 3], one could try to prove it by combining the results of [9] with the extension theorem of equivariant maps in [11]. We take here a different approach using much more elementary methods than [9] and getting some explicit information about the boundary structure along the way.

My original idea was to prove Proposition 4.1 by use of a monotonicity property of $\Psi$ and then use [11] to extend $\Psi$ from $\mathfrak{a}$ to $\mathfrak{p}$. This got essentially simplified 
by J. Faraut's observation that $\Psi$ is the gradient of $\varphi$ and therefore it is possible to use the method of [4, Prop 1.3.4] (which is a reproduction of a proof in [14]). This observation also makes [11] almost superfluous: we will need only a very small part of the argument of [11].

In the following we fix a set $E_{0} \subseteq \Pi$ as in Section 1 and an element $H_{0} \in$ $\mathfrak{a}\left(E_{0}\right)^{+}$. The condition (assumed) that $E_{0}$ contains no component of $\Pi$ means that the orbit $W \cdot H_{0}$ spans $\mathfrak{a}$ as a linear space. We denote by $C_{\mathfrak{a}}$ the convex hull of $W \cdot H_{0}$, by $C_{\mathfrak{a}}$ its interior. Similarly, $C_{\mathfrak{p}}$ will be the convex hull of $K \cdot H_{0}$ and


form. We denote by $\nabla_{\mathfrak{p}}, \nabla_{\mathfrak{a}}$ the gradient with respect to it on $\mathfrak{p}$ resp. on $\mathfrak{a}$.

On $\mathfrak{a}$ we define the function $\varphi$ by

$$
\varphi(H)=\sum_{s \in W} e^{\left(s H_{0} \mid H\right)}
$$

and we define the map $\Psi: \mathfrak{a} \rightarrow \mathfrak{a}$ by $\Psi=\nabla_{\mathfrak{a}} \log \varphi$, i.e.

$$
\Psi(H)=\frac{1}{\sum_{s \in W} e^{\left(s H_{0} \mid H\right)}} \sum_{s \in W} e^{\left(s H_{0} \mid H\right)} s H_{0} .
$$

( $\Psi$ is a direct explicit expression for the moment map also used by Ji [9].) Clearly, $\varphi$ is real analytic and $W$-invariant. By a version of Chevalley's theorem it extends to a $K$-invariant real analytic function $\tilde{\varphi}$ on $\mathfrak{p}$. The gradient $\Psi$ of $\log \varphi$ is then $W$-equivariant; similarly $\tilde{\Psi}=\nabla_{\mathfrak{p}} \log \tilde{\varphi}$ is $K$-equivariant as a map $\mathfrak{p} \rightarrow \mathfrak{p}$. At points of $\mathfrak{a}, \nabla_{\mathfrak{p}} \log \tilde{\varphi}$ is the same as $\nabla_{\mathfrak{a}} \log \varphi$ because $\log \tilde{\varphi}$ is constant on $K$-orbits and those are orthogonal to $\mathfrak{a}$. (With more detail this is in [11]; it is the only part of [11] we have to use.) So, we have $\tilde{\Psi}_{\mid \mathfrak{a}}=\Psi$, i.e. $\tilde{\Psi}$ is an extension of $\Psi$.

Proposition 4.1. $\Psi$ is a real analytic diffeomorphism of $\mathfrak{a}$ onto $\stackrel{\circ}{\mathfrak{a}}_{\text {. }}$.

Proof. Let $H^{\prime} \in \stackrel{\circ}{\mathfrak{a}}_{\mathfrak{a}}$. We show that it is the image of a unique $H \in \mathfrak{a}$ by showing that the function

$$
f(H)=\log \varphi(H)-\left(H^{\prime} \mid H\right)
$$

is (i) strictly convex, and (ii) tends to $\infty$ as $H \rightarrow \infty$ in $\mathfrak{a}$. (Then it has a unique minimum, so there is a unique $H$ where $\Psi(H)-H^{\prime}=\nabla f(H)=0$.)

Writing $D_{H_{1}}$ for the directional derivative we have for any $H_{1} \in \mathfrak{a}$,

$$
\begin{aligned}
D_{H_{1}}^{2} f(H) & =\frac{1}{\varphi(H)^{2}}\left(\varphi(H) D_{H_{1}}^{2} \varphi(H)-\left(D_{H_{1}} \varphi(H)\right)^{2}\right) \\
& =\frac{1}{\varphi(H)^{2}}\left(\sum_{s \in W} e^{\left(s H_{0} \mid H\right)} \sum_{s \in W} e^{\left(s H_{0} \mid H\right)}\left(s H_{0} \mid H_{1}\right)^{2}-\left(\sum_{s \in W} e^{\left(s H_{0} \mid H\right)}\left(s H_{0} \mid H_{1}\right)\right)^{2}\right) .
\end{aligned}
$$


By the Schwarz inequality this is positive since the $\left(s H_{0} \mid H_{1}\right)(s \in W)$ cannot be independent of $s$. This proves (i).

For (ii), when $H \neq 0$ and $t \in \mathbb{R}$ we can write

$$
f(t H)=\log \sum_{s \in W} e^{t\left(\left(s H_{0} \mid H\right)-\left(H^{\prime} \mid H\right)\right)} .
$$

Since $H^{\prime}$ is a strict convex combination of the $s H_{0}(s \in W)$, there is some $s$ such that $\left(s H_{0} \mid H\right)-\left(H^{\prime} \mid H\right)>0$. Therefore, $\lim _{t \rightarrow \infty} f(t H)=\infty$, proving (ii).

Finally we note that image of $\Psi$ must be contained in $\stackrel{\circ}{C}_{\mathfrak{a}}$ by (4.2).

REMARK 2. $\Psi$ maps $\mathfrak{a}(E)$ to $\mathfrak{a}(E)$ and $\mathfrak{a}(E)^{+}$to $\mathfrak{a}(E)^{+}$for every $E \subseteq \Pi$.

Proof. Writing $W^{E}$ for the subgroup of $W$ generated by the reflections in the roots $\lambda \in E$, the fixed set of $W^{E}$ is exactly $\mathfrak{a}(E)$. So, for $H \in \mathfrak{a}(E), \Psi(H)=$ $\Psi(s H)=s \Psi(H)$ is in $\mathfrak{a}(E)$. Since it preserves each $\mathfrak{a}(E), \Psi$ maps Weyl chambers to Weyl chambers. If the defining element $H_{0}$ of $\Psi_{H_{0}}=\Psi$ is in $\mathfrak{a}^{+}$, then (4.2) shows that $\Psi\left(t H_{0}\right)$ is near $H_{0}$ for large $t$, by $\left(H_{0} \mid H_{0}\right)>\left(s H_{0} \mid H_{0}\right),(s \neq e)$. So $\Psi$ preserves $\mathfrak{a}^{+}$. Otherwise, we write $H_{0}=\lim H_{n}$ with $H_{n} \in \mathfrak{a}^{+}$. Then $\Psi=\lim \Psi_{H_{n}}$ maps chambers to chambers and each $\Psi_{H_{n}}$ preserves $\mathfrak{a}^{+}$. So, $\Psi$ again preserves $\mathfrak{a}^{+}$. But then it also preserves the faces $\mathfrak{a}(E)^{+}$.

Proposition 4.2. $\tilde{\Psi}$ is a real analytic diffeomorphism of $\mathfrak{p}$ onto $\stackrel{\circ}{C}_{\mathfrak{p}}=K \cdot \stackrel{\circ}{C}_{\mathfrak{a}}$

Proof. $\overline{\mathfrak{a}}^{+}$is a fundamental domain for $W$ in $\mathfrak{a}$ and is the disjoint union of the $\mathfrak{a}(E)^{+},(E \subseteq \Pi)$. So, every point of $\mathfrak{p}$ has a representation $k \cdot \exp H$ with a unique $H$ in some $\mathfrak{a}(E)^{+}$and with $k \in K$ unique modulo $M_{K}(E)$. From the preceding Remark it is then immediate that $\tilde{\Psi}$ is one-to-one onto its image $K \cdot \stackrel{\circ}{C_{\mathfrak{a}}}$.

To see that $K \cdot \stackrel{\circ}{C}_{\mathfrak{a}}=\stackrel{\circ}{C}_{\mathfrak{p}}$ we note that $W \cdot H_{0} \subseteq K \cdot H_{0}$, implies $C_{\mathfrak{a}} \subseteq C_{\mathfrak{p}}$ and

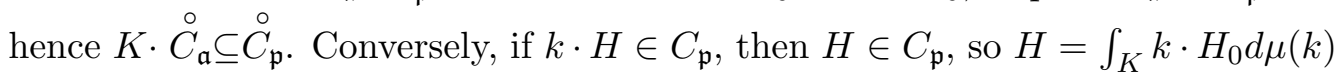
with some probability measure. Hence $H=\operatorname{pr}_{\mathfrak{a}} H=\int_{K} \operatorname{pr}_{\mathfrak{a}}\left(k \cdot H_{0}\right) d \mu(k)$, where $\operatorname{pr}_{\mathfrak{a}}$ denotes orthogonal projection onto $\mathfrak{a}$. By the easy part of Kostant's convexity theorem (see [7, p. 473]), $\operatorname{pr}_{\mathfrak{a}}\left(k \cdot H_{0}\right) \in C_{\mathfrak{a}}$. So, $H \in C_{\mathfrak{a}}, k \cdot H \in K \cdot C_{\mathfrak{a}}$, and $C_{\mathfrak{p}} \subseteq K \cdot C_{\mathfrak{a}}$.

Next we give a description of the boundary structure of $C_{\mathfrak{a}}$. Detailed proofs of our statements are in [3, Thm. 3.1], the proofs can also be based on [7, Lemma 8.3, p. 459] and the standard properties of root systems (e.g. [2, pp. $277-284]$ ).

$\mathfrak{a}^{+}$is a polyhedral cone whose open faces are the $\mathfrak{a}(E)^{+}(E \subseteq \Pi)$. We denote by ${ }^{+} \mathfrak{a}$ the open dual cone, i.e. the set $\left\{H \in \mathfrak{a} \mid\left(H^{\prime} \mid H\right)>0, \forall H^{\prime} \in \mathfrak{a}^{+}\right\}$. In each subalgebra $\mathfrak{a}^{E}$ we similarly define $\mathfrak{a}^{E+}$ and ${ }^{+} \mathfrak{a}^{E}$. The open faces of ${ }^{+} \mathfrak{a}$ are the 
${ }^{+} \mathfrak{a}^{E} ;{ }^{+} \overline{\mathfrak{a}}^{E}$ is the convex cone spanned by the $H_{\lambda}(\lambda \in E)$, where $H_{\lambda}$ denotes the element of $\mathfrak{a}$ such that $\lambda(H)=\left(H_{\lambda} \mid H\right), \forall H \in \mathfrak{a}$. Each closed face of $C_{\mathfrak{a}}$ that meets $\overline{\mathfrak{a}}^{+}$is contained in $H_{0}-{ }^{+} \overline{\mathfrak{a}}^{E}$ for some $E$ (for a short proof see [7, p. 459]).

Let now $E$ be $E_{0}$ - connected. We write $H_{0}=H_{0, E}+H_{0}^{E}$ following the decomposition $\mathfrak{a}=\mathfrak{a}(E)+\mathfrak{a}^{E}$. We have $W^{E} \cdot H_{0}=H_{0, E}+W^{E} \cdot H_{0}^{E}$, and $W^{E} \cdot H_{0}^{E}$ spans $\mathfrak{a}^{E}$ (since every component of $E$ contains some $\lambda \in \Pi$ with $\lambda\left(H_{0}^{E}\right) \neq 0$ due to the $E_{0}$-connectedness of $E$ ). Note that by $W^{E^{\prime}}=W^{E} \times W^{E^{\prime \prime}}$ we also have $H_{0, E} \in \mathfrak{a}\left(E^{\prime}\right)$ and $W^{E^{\prime}} \cdot H_{0}=W^{E} \cdot H_{0}$.

We denote by $C_{\mathfrak{a}}^{E}$ the convex hull of $W^{E} \cdot H_{0}^{E}$; this is a closed domain in the subspace $\mathfrak{a}^{E}$. The supporting hyperplane of $C_{\mathfrak{a}}$ orthogonal to $H_{0}^{E}$ meets $C_{\mathfrak{a}}$ exactly in $H_{0, E}+C_{\mathfrak{a}}^{E}$. So the $H_{0, E}+C_{\mathfrak{a}}^{E}$ are just the open faces of $C_{\mathfrak{a}}$ meeting $\overline{\mathfrak{a}}^{+}$; the other faces are $W$ - images of these.

We proceed to the boundary structure of $C_{\mathfrak{p}}$. First we note that Proposition 4.1 and 4.2 also hold for the symmetric space $X^{E} \cong M(E) / M_{K}(E)$ in place of $X$. So, for each $E_{0}$-connected $E$ there is an analytic diffeomorphism $\Psi^{E}: \mathfrak{a}^{E} \rightarrow C_{\mathfrak{a}}^{E}$ which extends to $\tilde{\Psi}^{E}: \mathfrak{p}^{E} \rightarrow \stackrel{\circ}{E}$ where $C_{\mathfrak{p}}^{E}$ denotes the relative interior of the convex hull $C_{\mathfrak{p}}^{E}$ of $M_{K}(E) \cdot H_{0}^{E}$. This is a domain in $\mathfrak{p}^{E}$, and we also have $C_{\mathfrak{p}}^{E}=M_{K}(E) \cdot C_{\mathfrak{a}}^{E}$.

Using the decomposition $H_{0}=H_{0, E}+H_{0}^{E}$ and the fact that $M_{K}(E)$ is a group of orthogonal linear transformations fixing $H_{0, E}$ it follows that the convex hull of $M_{K}(E) \cdot H_{0}$ is $H_{0, E}+C_{\mathfrak{p}}^{E}$ and that this is a closed face of $C_{\mathfrak{p}}$. The boundary of $C_{\mathfrak{p}}$ is partitioned into open faces which are of the form $k \cdot\left(H_{0}+C_{\mathfrak{p}}^{E}\right)$ with $E_{0}$-connected $E$ and $k \in K$ unique modulo $M_{K}\left(E^{\prime}\right)$.

After these remarks we can prove the main result of this Section.

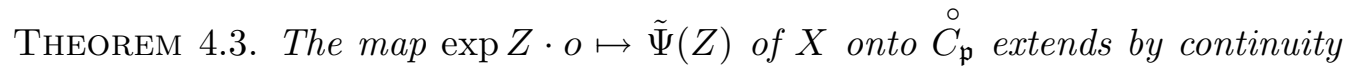
to a $K$-equivariant homeomorphism of $\bar{X}\left(E_{0}\right)$ onto $C_{\mathfrak{p}}$. This extension maps the boundary components onto the open faces of $C_{\mathfrak{p}}$ and is explicitly given by $k \cdot \iota_{E}(\exp Y \cdot o) \mapsto k \cdot\left(H_{0, E}+\tilde{\Psi}^{E}(Y)\right),\left(k \in K, Y \in \mathfrak{p}^{E}\right)$.

Proof. We take an arbitrary sequence $\left\{x_{n}\right\}$ in $X$ tending to a boundary point in $\bar{X}\left(E_{0}\right)$, i.e. we take $x_{n}=k_{n} \exp H_{n} \cdot o\left(k_{n} \in K, H_{n} \in \overline{\mathfrak{a}}^{+}\right)$converging to some $k \cdot \iota_{E}\left(\exp H^{E} \cdot o\right)$. Here $k_{n}$ can be chosen so that $\lim k_{n}=k$, and by $K$-equivariance we may assume $k=e$. The convergence means that $\lim \lambda\left(H_{n}\right)=\lambda(H)$ for $\lambda \in E$, and $\lim \lambda\left(H_{n}\right)=\infty$ for $\lambda \in \Pi-E^{\prime}$ (cf. the Satake axioms).

The image of $x_{n}$ is $k_{n} \Psi\left(H_{n}\right)$, we have to show that this converges to $H_{0, E}+$ $\Psi^{E}\left(H^{E}\right)$. For this we decompose $H_{n}$ as $H_{n, E}+H_{n}^{E}$ and look at the expression (4.2) 
of $\Psi\left(H_{n}\right)$. The coefficients are of the form $e^{\left(s H_{0} \mid H_{n, E}\right)} e^{\left(s H_{0} \mid H_{n}^{E}\right)}$. The second factor is bounded, since $\lim H_{n}^{E}=H^{E}$. The first factor, for $s \in W^{E^{\prime}}$, is independent of $s$. For $s \notin W^{E^{\prime}}$ it is of smaller order of magnitude than $e^{\left(H_{0} \mid H_{n}\right)}$ as $n \rightarrow \infty$ : In fact, $H_{0}-s_{0} H_{0}=\sum_{\Pi} p_{\lambda} H_{\lambda}$ with $p_{\lambda} \geq 0$ since it is in ${ }^{+} \mathfrak{a}$. It is not in $\mathfrak{a}^{E}$, so $p_{\lambda}>0$ for some $\lambda \notin E^{\prime}$. This implies that $\lim \left(H_{0}-s H_{0} \mid H_{n}\right)=\infty$.

So, in the limit all terms with $s \notin W^{E^{\prime}}$ drop out and the factor $e^{\left(H_{0} \mid H_{n, E}\right)}$ cancels giving

$$
\frac{1}{\sum_{s \in W^{E^{\prime}}} e^{\left(s H_{0}^{E} \mid H^{E}\right)}} \sum_{s \in W^{E^{\prime}}} e^{\left(s H_{0}^{E} \mid H^{E}\right)}\left(H_{0, E}+s H_{0}^{E}\right) .
$$

Since $W^{E^{\prime}}=W^{E} \times W^{E^{\prime \prime}}$ and $W^{E^{\prime \prime}}$ fixes $H_{0}^{E}$, the value of this expression remains the same if we take the sums over $W^{E}$. This proves that $\lim k_{n} \Psi\left(H_{n}\right)=H_{0, E}+$ $\Psi^{E}\left(H^{E}\right)$.

To complete the proof of the continuity of the extension of $\tilde{\Psi}$ we also have to consider convergent sequences contained in the boundary $\bar{X}\left(E_{0}\right)$ and their images under the extended map. This can be done by looking at sequences in one boundary component at a time, and making use of the hereditary structure of the compactification (a boundary component of a boundary component of $X$ is again a boundary component of $X)$. For a sequence $x_{n}$ contained in $k \cdot \iota_{D}\left(X^{D}\right)$ and converging to a point in $k^{\prime} \cdot \iota_{E}\left(X^{E}\right)$, the same argument works as above. We omit the details.

At this point we have a continuous $K$-equivariant extension of our map. It is onto $C_{\mathfrak{p}}$ because $C_{\mathfrak{p}}$ is the disjoint union of $\stackrel{\circ}{C_{\mathfrak{p}}}$ and its open faces $k \cdot\left(H_{0, E}+\stackrel{\circ}{C_{\mathfrak{p}}^{E}}\right)$ and all points of these arise in the form $k^{\prime} \cdot\left(H_{0, E}+\Psi^{E}\left(H^{E}\right)\right)$. It is also one - to - one because $\tilde{\Psi}^{E}$ is by (the analogue of) Proposition 4.2 and because the components $k \cdot \iota_{E}\left(X^{E}\right)$ and the faces $k \cdot\left(H_{0, E} C_{\mathfrak{p}}^{E}\right)\left(k \in K / M_{E}\left(E^{\prime}\right)\right)$ correspond to each other in a one - to - one way. Therefore, by compactness, it is a homeomorphism.

REMARK 3. The Harish-Chandra embedding of Hermitian symmetric spaces as bounded domains in $\mathbb{C}^{n}$ is a special instance of our realizations $\stackrel{\circ}{C}_{\mathfrak{p}}$. In fact, assuming as we may that $X$ is irreducible Hermitian symmetric, we see from [8, Thm. 4.10, p. 460] that $\mathfrak{a}$ has an orthonormal basis $H_{i}(1 \leq i \leq \ell)$ such that $H=\sum t_{i} H_{i}$ is in $\mathfrak{a}^{+}$iff $t_{1}>t_{2}>\cdots>t_{\ell}>0$, and $W$ consists of all signed permutations of the $H_{i}$. (We write $H_{i}$ for $H_{\gamma_{i}}$ of [8].) We take $H_{0}$ on the edge $t_{1}=t_{2}=\cdots=t_{\ell}$ of $\overline{\mathfrak{a}}^{+}$; up to an unessential homothety we may assume $H_{0}=\sum H_{i}$. Now the $W$ - orbit of $H_{0}$ consists of all $\sum \varepsilon_{i} H_{i}$ with $\varepsilon_{i}= \pm 1$. So,

$$
\varphi(H)=\sum e^{\sum \varepsilon_{i} t_{i}}=\prod\left(e^{t_{i}}+e^{-t_{i}}\right)=2^{\ell} \prod \cosh t_{i}
$$


and

$$
\Psi(H)=\nabla \log \varphi(H)=\sum\left(\tanh t_{i}\right) H_{i} .
$$

Comparing with $[8$, pp. $454-5]$ we see that $\tilde{\Psi}$ coincides with the Harish-Chandra imbedding.

\section{REFERENCES}

[1] A. Borel and L. Ji, Compactifications of symmetric and locally symmetric spaces, Math. Research Letters 9 (2002), 725 - 739.

[2] N. Bourbaki, Groupes et algèbres de Lie, Chapters 4,5 et 6, Hermann, Paris, 1968.

[3] W. A. Casselman, Geometric rationality of Satake compactifications, to appear in Algebraic groups and related subjects, ed. by G. I. Lehrer et al., a volume of Australian Mathematical Society Lecture series in honor of R. W. Richardson.

[4] J. Faraut and A. Korányi, Analysis on symmetric cones, Oxford University Press, 1994.

[5] H. Furstenberg, A Poisson formula for semi-simple Lie groups, Ann. Math. 77 (1963), 335 $-386$.

[6] Y. Guivarc'h, L. Ji and J. C. Taylor, Compactification of symmetric spaces, Birkhauser, 1998.

[7] S. Helgason, Groups and geometric analysis, Acad. press 1984, Amer. Math. Soc. 2000.

[8] S. Helgason, Geometric analysis on symmetric spaces, Amer. Math. Soc. 1994.

[9] L. Ji, Satake and Martin compactifications of symmetric spaces are topological balls, Math. Res. Letters 4 (1997), 79 - 89.

[10] A. Korányi, Poisson integrals and boundary components of symmetric spaces, Inv. Math. 34 (1976), 19 - 35.

[11] A. Korányi and R. Szőke, On Weyl group equivariant maps, to appear.

[12] C. C. Moore, Compactifications of symmetric spaces I, II, Amer. J. Math. 86 (1964), 201 $218,358-378$.

[13] I. Satake, On representations and compactifications of symmetric Riemannian spaces, Ann. Math. 71 (1960), 77 - 110.

[14] E. B. Vinberg, The theory of convex homogeneous cones, Trudy Mosk. Math. Obs. 12 (1963), 303 - 358; Trans. Moscow Math. Soc. 12 (1963), 303 - 358.

Adam Korányi

Lehman College

The City University of New York

Bronx, NY 10468

E-mail: adam.koranyi@lehman.cuny.edu 